\title{
Predictive Model of Graphene Based Polymer Nanocomposites: Electrical Performance
}

\author{
Asimina $\operatorname{Manta}^{1} \cdot$ Matthieu Gresil $^{1,2} \cdot$ Constantinos Soutis $^{2}$
}

Received: 2 September 2016 / Accepted: 30 October 2016 / Published online: 18 November 2016

C The Author(s) 2016. This article is published with open access at Springerlink.com

\begin{abstract}
In this computational work, a new simulation tool on the graphene/polymer nanocomposites electrical response is developed based on the finite element method (FEM). This approach is built on the multi-scale multi-physics format, consisting of a unit cell and a representative volume element (RVE). The FE methodology is proven to be a reliable and flexible tool on the simulation of the electrical response without inducing the complexity of raw programming codes, while it is able to model any geometry, thus the response of any component. This characteristic is supported by its ability in preliminary stage to predict accurately the percolation threshold of experimental material structures and its sensitivity on the effect of different manufacturing methodologies. Especially, the percolation threshold of two material structures of the same constituents (PVDF/Graphene) prepared with different methods was predicted highlighting the effect of the material preparation on the filler distribution, percolation probability and percolation threshold. The assumption of the random filler distribution was proven to be efficient on modelling material structures obtained by solution methods, while the through-the -thickness normal particle distribution was more appropriate for nanocomposites constructed by film hot-pressing. Moreover, the parametrical analysis examine the effect of each parameter on the variables of the percolation law. These graphs could be used as a preliminary design tool for more effective material system manufacturing.
\end{abstract}

Matthieu Gresil

matthieu.gresil@manchester.ac.uk

Asimina Manta

asimina.manta@postgrad.manchester.ac.uk

Constantinos Soutis

constantinos.soutis@manchester.ac.uk

1 i-composites lab, School of Materials, University of Manchester, James Lighthill Building, Sackville Street, Manchester M13 9PL, UK

2 Aerospace Research Institute, Faculty of Engineering and Physical Sciences, University of Manchester, James Lighthill Building, Sackville Street, Manchester M13 9PL, UK 
Keywords Graphene $\cdot$ Polymer · Finite element analysis $\cdot$ Electrical conductivity ·

Nanocomposites $\cdot$ Computational methods $\cdot$ Multi-scale

\section{Introduction}

Conductive polymers have been extensively studied for their potential applications in light emitting devices, batteries, electromagnetic shielding, and piezoresistive sensors. At first, carbon black [1, 2], metallic powder [3-5], polyaniline [6] and graphite [7] were used as electrical reinforcement in polymer, but high concentration was necessary to achieve the percolation threshold which endangered the mechanical properties of the nanocomposites due to the formation of agglomerations. Later, several researchers proposed polymer nanocomposites reinforced with graphene nanoparticles and its derivatives (expanded graphite, graphene nanoplatelets, graphite oxide, functionalized graphene/expanded graphite), which are able to form more stable 3D conductive networks in lower volume content as a consequence of their high aspect ratio (AR-ratio of main particle dimension to minor one) [8-15]. Comparing the available experimental data in terms of percolation threshold varied by the filler aspect ratio, it could be easily noted its high dependence on the nanocomposite's manufacturing process (it affects the filler distribution and orientation as well as the formation of agglomerations), while for a given production methodology and materials constituents, the percolation threshold is not a deterministic quantity but a probabilistic one. This notice could lead to the conclusion that any percolation threshold stated is a misleading achievement if the material characteristics, manufacturing process and the probability of conductance are not clearly mentioned. In this field, the simulation approaches of the nanocomposite's electrical response could be a useful prediction tool on the full electrical characterisation of these materials taking into account the probabilistic nature of their response, since huge statistical samples could be studied much quicker and cheaper than following an experimental procedure.

\subsection{Electrical Simulation Models}

Despite the high financial and time cost of material preparation and experimental work, there are a few computational models predicting the electrical response of graphene based polymer nanocomposites. In the literature, the electrical simulation models are divided into two main categories- the percolation threshold models and the full electrical response. As far as the percolation models are concerned, there is a wide variety of proposed methodologies taking into account the filler shape, aspect ratio, tunnelling distance, overlapping, and the formation of agglomerations. Oskouyi et al. [16] apply the Monte Carlo method to model the percolation threshold for disk-shaped fillers, simulating the conductive network formed by inclusions like graphene nanoplatelets (GNP). Later, Ambrosetti et al. [17] conducted a numerical study to investigate a system's percolative properties consisting of hard oblate ellipsoids of revolution surrounded with soft penetrable shells. Since the previously mentioned computational works were focusing only on material systems with constant filler material and geometrical properties, Otten et al. [18] developed an analytical approach to investigate the percolation behaviour of polydisperse nanofillers of platelet-based composites. However, their modelling approach was subject to certain limitations, that is, the platelet thickness and the tunnelling decay length have to be of the same order of magnitude, and the diameter of the disk-like fillers needs to be much larger than the disk thickness. In terms of exploring the effect of filler overlapping and agglomerations, Xia et al. 
[19] proposed a computational methodology under which it was possible to predict the percolation threshold for identical ellipses with the overlapping effect for a $2 \mathrm{D}$ structure, while Vovchenko et al. [20] predicted the percolation threshold of composites filled with intersecting circular discs in a 3D structure. Besides, the conventional Monte Carlo approaches to predict the percolation threshold for materials reinforced with 2D particles, Mathew et al. [21] conducted a Monte Carlo study on the percolation of hard platelets in a 3D continuum system considering the rate of order in the microstructure, proving its effect on the percolation threshold with the employment of isotropic-nematic (IN) transition.

Although there is a satisfactory number of numerical methods predicting the percolation threshold of nanocomposites doped with fillers of various shapes and sizes, there are a few simulation tools on the prediction of the full electrical response of these nanocomposites. At first, Hicks et al. [22] developed a tunnelling-percolation model to investigate electrical transport in graphene based nanocomposites, covering the need of a suitable model to predict the full electrical response of semiconducting 2D element reinforced materials. This model, however, is applicable only to rectangle shaped nanoparticles forming $2 \mathrm{D}$ networks, while in common graphene reinforced nanocomposites, fillers exhibit a wide range of shapes, mainly circular-ellipsoid ones, and the conductive network formed is considered to be a 3D one. In a later work, Ambrosetti et al. [23] studied the electrical conductivity of an insulating matrix reinforced with conductive ellipsoids by assuming that an expected curve of electrical conductivity variation would be applied and finally being reduced in a geometrical form taking into account the inter-particle distance and the tunnelling distance. Oskouyi et al. [24] developed a 3D Monte Carlo model to study the percolation, conductivity, and piezoresistive behaviour of composites filled with randomly dispersed impenetrable conductive nano-disks. In this study, a Monte Carlo model was first developed to form a representative volume element (RVE) filled with randomly dispersed nano-platelet conductive inclusions. Then, a 3D finite element based resistors network model was used to analyse the conductivity behaviour of nano-platelet based conductive polymers. Finally, they developed a continuum model to determine the effective AC and DC electrical properties of graphene nanocomposites [25]. The proposed theory consisted of three major components, embodying the most fundamental characteristics of the graphene nanocomposites, i.e. percolation threshold, interface effects and additional contribution of electron hopping and micro capacitor structures to interfacial properties.

Knowing that, such numerical models are not easily applicable on industrial and engineering case study, as their employment is complicated and their results are limited to certain case. In this paper, a multi-scale multi-physics finite element model (FEM) is presented which would be easily applied to most cases, extended to more sophisticated material architecture in respect of being scientifically structured and proven. The current approach on multi scale modelling consists of the creation of a unit cell and a micro-scale nanocomposite model (Representative Volume Element RVE) on a commercial finite element modelling environment. The unit cell consists of a single rectangular or elliptical graphene plate surrounded by a thin layer of polymer. This polymer layer represents the inter-plate volume between successive graphene reinforcements, in which conduction phenomena (tunnelling effect, electron hopping) take place. The unit cell is loaded with a constant electric potential to compute the resistance matrix representing this system. The RVE is a rectangular block, on whose resistance matrix elements previously obtained through the unit cell is randomly distributed. This distribution represents the random position of graphene on the bulk volume of polymer nanocomposites. The orientation of graphene is simulated by the 3D random orientation of the corresponding element local coordinate system. 


\section{Finite Element Modelling}

\subsection{Unit Cell}

Similarly to the approach of hard core-penetrable shell has been assumed on several studies predicting the percolation threshold [17, 22], the unit cell consists of a single-layer graphene plate surrounded by a thin layer of polymer as shown in Fig. 1 (rectangular shape) and Fig. 2 (ellipsoid shape).

Three main geometrical parameters could be derived-graphene's plate shape and size (expressed by the Aspect Ratio) and the polymer layer thickness $d_{t}$. Graphene is modelled as an isotropic electrical conductive material with electrical conductivity of $\sigma=10^{7} \mathrm{~S} / \mathrm{m}$, while the polymer layer represents the volume between consecutive graphene, where the tunnelling effect phenomenon takes place. As a consequence, the conduction mechanism on polymer volume would be simulated by applying an exponentially varied resistivity $\rho_{\text {tunnel }}$ expressed by equation (1) in accordance to the findings of [26] for the electric tunnel effect between similar electrodes separated by a thin insulating film. The terms of the equation (1) are fully presented in Table 1.

$$
\rho_{\text {tunnel }}=\frac{h^{2}}{e^{2} \sqrt{2 m \lambda}} \exp \left(\frac{4 \pi d}{h} \sqrt{2 m \lambda}\right)
$$

Equation (1) describes the current flow between two conductive electrodes when they are separated by an insulating film, under the condition that the top of the energy gap of the insulator is above the Fermi level of the electrodes. The electronic current can flow through the insulating region between the two electrodes if: (i) the electrons in the electrodes have enough thermal energy to surmount the potential barrier; and (ii) the flow in the conduction band or the barrier is thin enough to permit its penetration by the electric tunnel effect. Knowing that the electrons' thermal energy could additionally contribute to the electric current, this effect was neglected requesting low-temperature conditions.

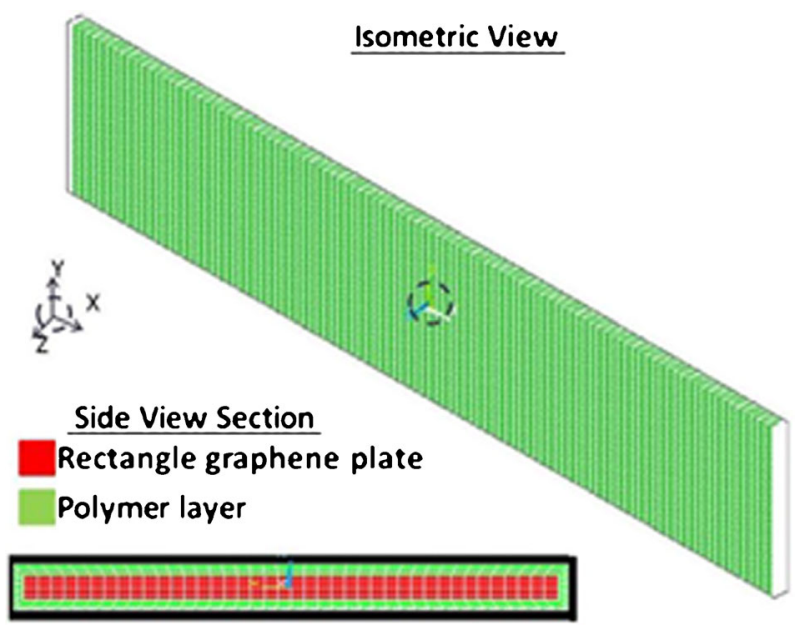

Fig. 1 Rectangular unit cell element model 
Fig. 2 Elliptical unit cell finite

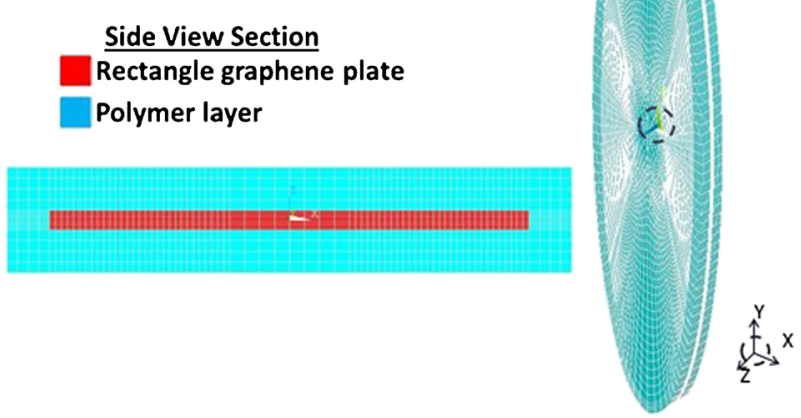

Since the conduction phenomena take place between two successive conductive particles, it is necessary to modify the application of the tunnelling resistivity in the unit cell, so as to simulate the contribution of a single plate to the conduction. In many studies, the cut-off distance (maximum inter-particle distance above this the tunnelling effect is eliminated) was considered around $2 \mathrm{~nm}$ [22-24], leading to the polymer thickness range between 0 and $1.0 \mathrm{~nm}$. Moreover, it is crucial to note that the architecture of graphene plates forms conductive paths inside the insulating polymer, which could be represented as a typical $2 \mathrm{D}$ or even $3 \mathrm{D}$ resistor network. As a consequence, if the resistance of each unit cell was used to from a network, the result would be to add the resistance of two graphene plates and the tunnelling resistance assigned to each plate for every pair of plates. However, due to the fact that the tunnelling resistance is an exponential function of tunnelling distance, the sum of assigned tunnelling resistance of each plate would not be equal to the actual resistance. This observation implied the need to modify the equation (1) by multiplying it with the equation (2), in order to simulate the actual tunnelling resistance when formed in a 3D real nanocomposite structures.

$$
\frac{R_{\text {tunnel }}\left(d_{1}+d_{2}\right)}{R_{\text {tunnel }}\left(d_{1}\right)+R_{\text {tunnel }}\left(d_{2}\right)}=\frac{d_{1}}{d_{1}+d_{2}} \exp \left(\frac{-4 \pi d_{2}}{h} \sqrt{2 m \lambda}\right)+\frac{d_{2}}{d_{1}+d_{2}} \exp \left(\frac{-4 \pi d_{1}}{h} \sqrt{2 m \lambda}\right)
$$

where $d_{1}$ and $d_{2}$ are the polymer thicknesses of two different unit cells named $\mathrm{UC}_{1}$ and $\mathrm{UC}_{2}$, $\mathrm{R}_{\text {tunnel }}\left(\mathrm{d}_{1}+\mathrm{d}_{2}\right)$ is the tunnelling resistance exhibited between the conductive particles $\mathrm{UC}_{1}$ and $\mathrm{UC}_{2}$ with total tunnelling distance $\mathrm{d}_{1}+\mathrm{d}_{2}$, and $\mathrm{R}_{\text {tunnel }}\left(\mathrm{d}_{1}\right)$ and $\mathrm{R}_{\text {tunnel }}\left(\mathrm{d}_{2}\right)$ are the tunnelling resistances exhibited in the polymer layer in each one of the unit cells $\mathrm{UC}_{1}$ and $\mathrm{UC}_{2}$, while $\mathrm{m}$ is the electron mass, $\mathrm{h}$ is the Plank's constant and $\lambda$ is the height of barrier.

Table 1 Tunnelling resistivity variables and parameters

\begin{tabular}{llll}
\hline Plank's constant & $\mathrm{h}$ & $\mathrm{m}^{2} \mathrm{~kg} / \mathrm{s}$ & $6.62607004 \cdot 10^{-34}$ \\
Electron charge & $\mathrm{e}$ & Coulomb & $1.60217662 \cdot 10^{-19}$ \\
Electron mass & $\mathrm{m}$ & $\mathrm{Kg}$ & $9.10938356 \cdot 10^{-31}$ \\
Height of barrier & $\lambda$ & $\mathrm{eV}$ & $0.5-2.5$ \\
Polymer layer thickness or 1/2Tunnelling distance & $\mathrm{d}_{\mathrm{t}}$ & $\mathrm{nm}$ & $0.0-1.0$ \\
\hline
\end{tabular}


In the proposed function (2), it is clear that the definition of the tunnelling resistivity applied on the unit cell would not be a deterministic variable but a stochastic one. This statistical feature, then, should be transferred to the next scale and be applied on the RVE implying complicated distributions on material properties. To eliminate this effect, an equivalent constant $\mathrm{C}$ in combination with the modified equation (3) of tunnelling resistivity is proposed.

$$
\rho_{\text {tunnel }}=C_{\text {equivalent }} \frac{h^{2}}{e^{2} \sqrt{2 m \lambda}} \exp \left(\frac{8 \pi d}{h} \sqrt{2 m \lambda}\right)
$$

The constant $\mathrm{C}$ would be given by equation (4), depicted in Fig. 3 and its equivalent value would be the result of the equation (5). The equivalent value of $C$ for each $d_{2}$ is presented in Table 2, where it is possible to conclude that assuming $\mathrm{C}$ being equal to 0.3 would be reasonable to represent each case.

$$
\begin{gathered}
R_{\text {tunnel }}\left(d_{1}+d_{2}\right)=C\left(R_{\text {tunnel }}\left(d_{1}\right) \exp \left(\frac{4 \pi d_{1}}{h} \sqrt{2 m \lambda}\right)+R_{\text {tunnel }}\left(d_{2}\right) \exp \left(\frac{4 \pi d_{2}}{h} \sqrt{2 m \lambda}\right)\right)(4) \\
C_{\text {equivalent }}\left(d_{\max }-d_{\min }\right)=\int_{d_{\min }}^{d_{\max }} C(\delta) d \delta
\end{gathered}
$$

This model was built using the commercial FEA program ANSYS 16.2. 3D 20-Node Hex couple-field solid element was used with its piezoresistive behaviour activated through the available key-option 101, while both graphene and polymer isotropic electrical behaviour was assumed. It is important to mention that the piezoresistive coupling induced by this key-option was neglected by not defining the piezoresistive matrix.

The values of the parameters used on the analysis of the unit cell are presented on the Tables 3 and 4, while the unit cell geometrical parameters are schematically presented in Fig. 4.

As far as the single-layer graphene thickness is concerned, reports from literature show a distinct variation of the measured thickness of single layer graphene, which could be attributed

Fig. 3 Variation of constant $\mathrm{C}$ in function of polymer thickness surrounding graphene layer, where $\mathrm{d}_{1}$ and $\mathrm{d}_{2}$ are the polymer thicknesses of two different unit cells named $\mathrm{UC}_{1}$ and $\mathrm{UC}_{2}$ to be combined to form a part of a network

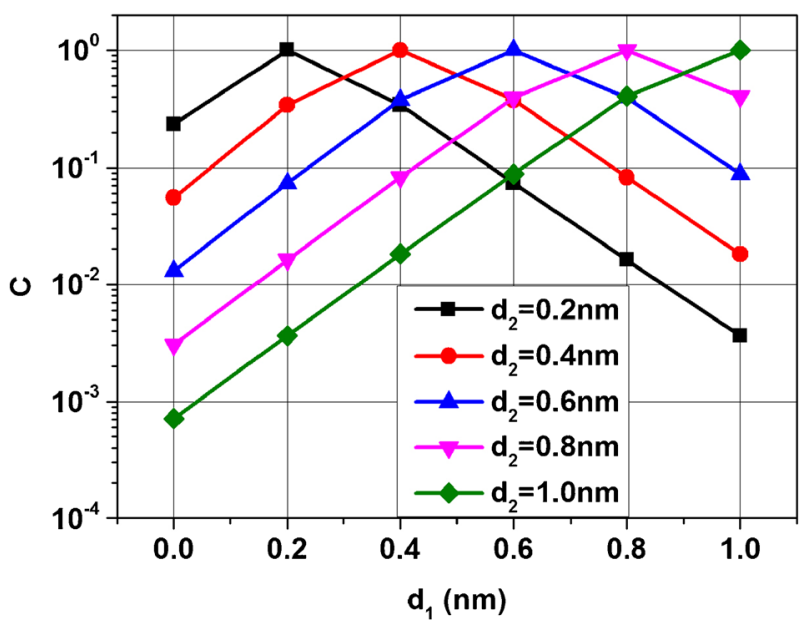


Table $2 \mathrm{C}_{\text {equivalent }}$ in function of $\mathrm{d}_{2}$

\begin{tabular}{ll}
\hline $\mathrm{d}_{2}(\mathrm{~nm})$ & $\mathrm{C}_{\text {equivalent }}$ \\
\hline 0.2 & 0.37 \\
0.4 & 0.37 \\
0.6 & 0.38 \\
0.8 & 0.34 \\
1.0 & 0.20 \\
\hline
\end{tabular}

to the measurement method and the graphene purity [27-34]. It has been stated that the thickness of a single-layer could range between $0.3 \mathrm{~nm}$ and $1.6 \mathrm{~nm}$. However, in accordance with the thickness measured by the imaging mechanism of tapping mechanism AFM, it was estimated around $0.4 \mathrm{~nm}$ [35]. It has been shown from SEM images that graphene particles could have several shapes, which are related to the manufacturing of the graphene and the breakage of the sheets during the homogenisation/dispersion of graphene/polymer materials. Generally, taking into account that the most common shapes able to describe a 2D structure are the rectangle and the ellipse as well as that these shapes have been considered for the prediction of percolation threshold. These two shapes are chosen for exploring their effect on the conduction of graphene/polymer nanocomposites.

In this analysis, the height of barrier (i.e. energy state of the insulating material to be reached by the electrons so as to conduction occur) is set as a parameter its effect needs to be explored, and not as a constant variable. The height of barrier usually takes values between 0.5 and $2.5 \mathrm{eV}[28,29]$ and is dependent of the polymer structure (crystalline, amorphous structure, crosslinking grade), and the chemical composition in the inter-particle region. In the literature, there is one major technique on height of barrier experimental calculation reported [28-31]. A specimen, consisting of two conductive electrodes separated by a thin layer of the insulating material of interest, is loaded under direct current. The current-voltage (I-V) response is plotted and fitted with the theoretical equation describing the junction.

Unit cell is loaded in the longitudinal, transverse and through thickness direction by a constant voltage, simulating DC loading and resulting to the calculation of the resistance matrix by the use of Ohm's law (Tables 5 and 6).

\subsection{Representative Volume Element (RVE)}

The nanocomposite materials is modelled by a square block of side equal to multiplied $\mathrm{b}(\mathrm{k} \cdot \mathrm{b})$ and thickness of multiplied a $(\mathrm{k} \cdot \mathrm{a})$, as shown in Fig. 5. The number of graphene seeds on the block is defined by equations $(6,7)$ as a function of nanocomposites volume fraction and graphene's geometry. The number of elements representing each graphene layer $(\gamma)$ would

Table 3 Graphene geometrical parameters

\begin{tabular}{llll}
\hline Graphene shape & & Rectangular & Elliptical \\
\hline Graphene plate thickness & $\mathrm{t}(\mathrm{nm})$ & 0.45 & 0.45 \\
Graphene minor side dimension & $\mathrm{a}(\mathrm{nm})$ & 10 & $2 \cdot 5.64$ \\
Aspect ratio & $\mathrm{AR}(-)$ & $1,5,10,50$ & $1,2,5,7,10$ \\
Graphene major side dimension & $\mathrm{b}(\mathrm{nm})$ & AR·a & AR·a \\
\hline
\end{tabular}


Table 4 Unit cell material properties

\begin{tabular}{llll}
\hline Material & Electrical behaviour & & \\
\hline Graphene & Electrical conductivity & $\mathrm{S} / \mathrm{m}$ & $10^{7}$ \\
Polymer & Tunnelling resistivity & $\Omega \mathrm{m}$ & Equation (3) \\
& C equivalent & - & 0.3 \\
& Height of barrier $(\lambda)$ & $\mathrm{eV}$ & $0.5-2.5$ \\
& $1 / 2$ Tunnelling distance $(\mathrm{d})$ & $\mathrm{nm}$ & $0.2-1.0$ \\
\hline
\end{tabular}

equal to the number of division in the direction of loading (eix) divided by the factor $\mathrm{k}$. As a consequence the number of elements representing graphene reinforcement total in the volume $\left(\mathrm{n}_{\mathrm{g}}\right)$ is defined by equation (8). The number of elements perpendicular to the loading direction (eiz) is given by equations $(9,10)$, while the number of elements through the block's thickness (eiy) is $e i z / A R$.

$$
n_{g}=\gamma n=n \frac{e i x}{k}
$$

The nanocomposite's architecture is modelled by the distribution of the graphene plates being enforced by choosing $n_{g}$ non-repeatable integer numbers. Each number indicates the element ID number in the FE model. Each selected element has the electrical properties resulted by the analysis of the unit cell. Especially, assuming DC linear electrical behaviour, the electrical response of the unit cell in each element is introduced in accordance to equations presented in Table 7. The tunnelling distance between two successive graphene particles is assigned by random choice obtained by the unit cell and related to a specific tunnelling distance. Finally, the orientation of graphene layers in the volume is modelled by the orientation of the local coordinate system with rotation angles of $T H X Y \in[0,90], T H Z Y \in[0$, 90] and $T H X Z \in[-90,90]$. The elements, which have not been chosen to represent graphene sheets, are simulated as pure insulating matrix with electrical resistivity of $10^{16} \Omega \mathrm{m}$. The RVE model was built up under the same principles as the unit cell one. The volume is loaded with constant voltage, while the result of the analysis is the reaction current. With the use of the Ohm's law and under the conduction of 50 separate analysis per case, the variation of electrical

\section{Polymer}

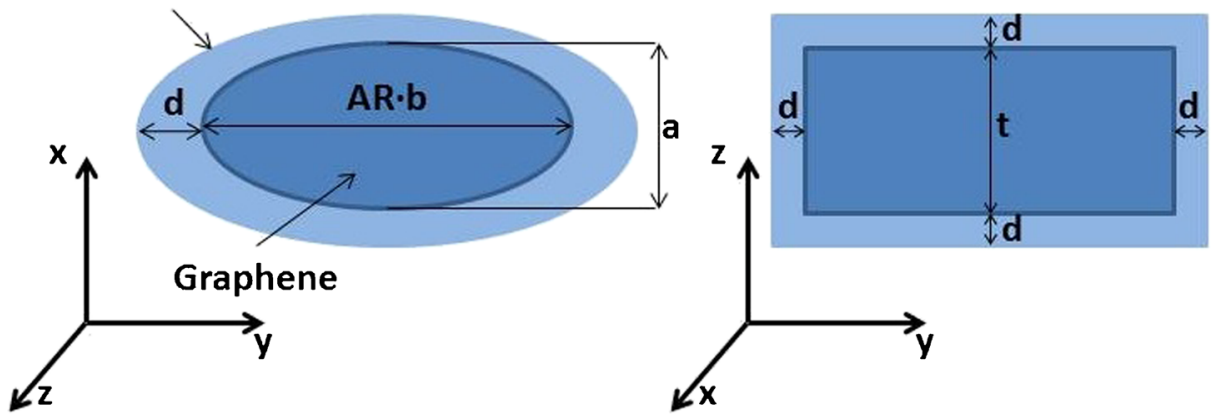

Fig. 4 Schematic presentation of the unit cell geometrical parameters 
Table 5 Equations describing the number of graphene seeds in respect of the graphene shape

Number of graphene seeds in respect of the graphene shape

\begin{tabular}{ll}
\hline Square - Rectangle & $n=V_{f} \frac{k^{3} A R a}{t}$ \\
Circle - Ellipse & $n=V_{f} \frac{k^{3} A R a}{\pi t}$
\end{tabular}

conductivity in respect of volume fraction is modelled, while in the probability function of percolation the inflection point is calculated to be the percolation threshold in accordance to the suggested procedure described in Ref. [21].

\section{Results}

\subsection{Unit Cell Analysis}

After the analysis of the unit cell in respect of all the parameters mentioned on Tables 3 and 4 , it is possible to analyse the effect of these features on the unit cell resistance, which represents the local resistance of the nanocomposites. The rise of the aspect ratio leads to significant decrease in the resistance. In longitudinal direction (x-direction), the resistance saturates to a constant value in function of the $1 / 2$ tunnelling distance for filler's aspect ratio being greater than 50, as it was fully studied in the [36]. Moreover, polymer and all the features controlling its response under any excitation (degree of polymerization, voids, humidity, temperature etc.) have serious effect on the electrical conductivity of a nanocomposite through the energy quantity of height of barrier appearing on the tunnelling resistivity. The effect of height barrier on the unit cell resistance in respect of tunnelling distance and for the case of rectangular graphene with aspect ratio of 1 and 5 is depicted in Fig. 6. An increase in the height of barrier results in the rise of the local resistance even for orders of magnitude. This increasing trend is common for every case of unit cell studied, while becomes more evident with the increase of tunnelling distance.

Finally, the effect of the shape of the graphene layer on the unit cell resistance is considered in respect of the direction and the tunnelling distance is depicted for all examined aspect ratios and height of barrier of $0.5 \mathrm{eV}$ [36]. It could be stated that ellipse shaped graphene sheets exhibit higher local resistance in the main axis directions than the rectangle shaped ones. However, for the case of the through thickness resistance, for the common aspect ratios ellipse and rectangle shaped graphene plates show the same electrical resistance. It is important to mention that for the purposes of comparison, the area of the rectangle shaped graphene layers is equal to that of the elliptical shaped graphene layers for each aspect ratio.

Table 6 Equations describing the number of elements on perpendicular to loading direction in respect of the graphene shape

Number of element on perpendicular to loading direction in respect of graphene shape

$\begin{array}{ll}\text { Square - Rectangle } & \text { eiz }=k A R \sqrt{\frac{a}{t}} \\ \text { Circle - Ellipse } & \text { eiz }=k A R \sqrt{\frac{a}{\pi t}}\end{array}$


Fig. 5 Representative volume element finite element model

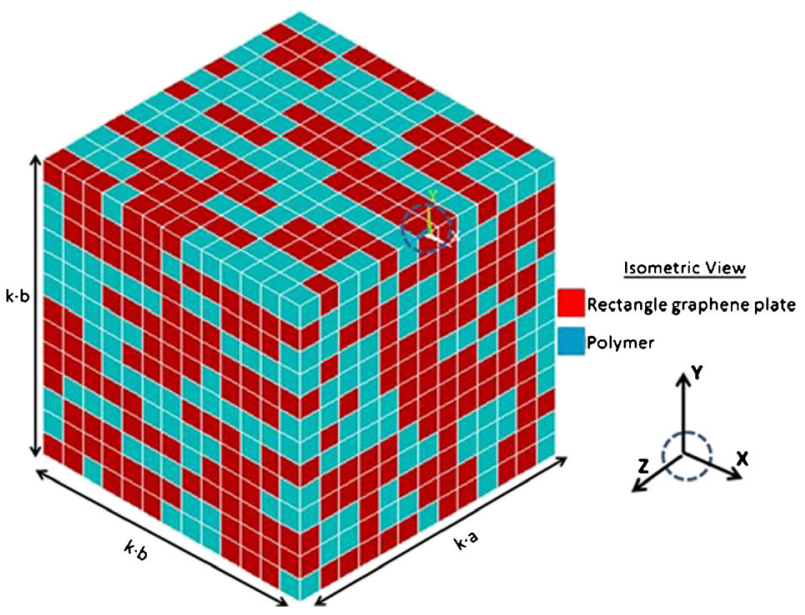

\subsection{RVE Analysis}

Since it is not practical to model infinite material and to eliminate the edge effect, it is important to conduct a convergence study on the size of the square block. The ratio of the sample's average electrical conductivity to its standard deviation has been considered to be the convergence parameter. As it could be noticed in Fig. 7, for $\mathrm{k}=5$ the sample characteristics have converged to a steady state, indicating this size as appropriate for our study.

The percolation probability, in accordance to the procedure suggested by the [21], is fitted by the function (14) and the inflection point of this function is considered to be the percolation threshold. In Fig. 8, the percolation probability, in respect of the volume fraction, is depicted for the case of rectangle graphene sheets and a height of barrier $\lambda=0.5 \mathrm{eV}$. The proposed fitting function is suitable to describe this data set achieving $99.999 \%$ accuracy on fitting process.

$$
P\left(V_{f}\right)=\frac{1}{2}\left(1+\tanh \left(A\left(V_{f}-B\right)\right)\right)
$$

As far as the effect of aspect ratio on the electrical conductivity in respect of the graphene volume fraction is concerned, the rise in the aspect ratio leads to a decrease on the volume fraction on which the onset of conductance occurs. In addition to this, for a constant value of volume fraction, the electrical conductivity increases with raising the aspect ratio. These results suggest that fillers with high aspect ratio are able to form more stable and efficient conductive

Table 7 Equations for the electrical resistivity introduced to the elements representing graphene particles in respect of the direction

Electrical resistivity distributed in the RVE in respect of the direction

\begin{tabular}{ll} 
X-axis & $\rho_{x}=R_{x}^{\text {unitcell } \frac{k^{2} A R a}{e i z^{2}}}$ \\
y-axis & $\rho_{y}=R_{y}^{\text {unitcell } \frac{k^{2} A R a}{e i x^{2}}}$ \\
z-axis & $\rho_{z}=R_{z}^{\text {unitcell }} \frac{k^{2} A R a}{e i x^{2}}$ \\
\hline
\end{tabular}




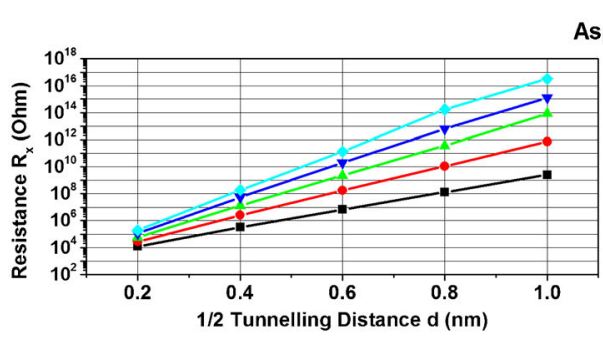

Aspect Ratio $=1$

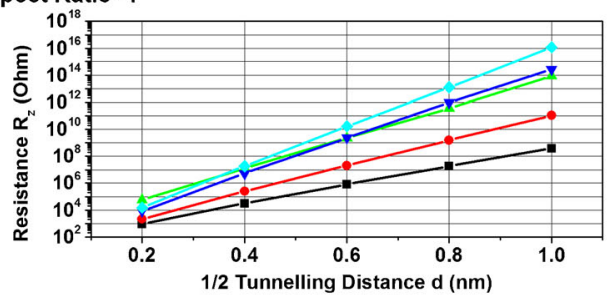

Aspect Ratio=5
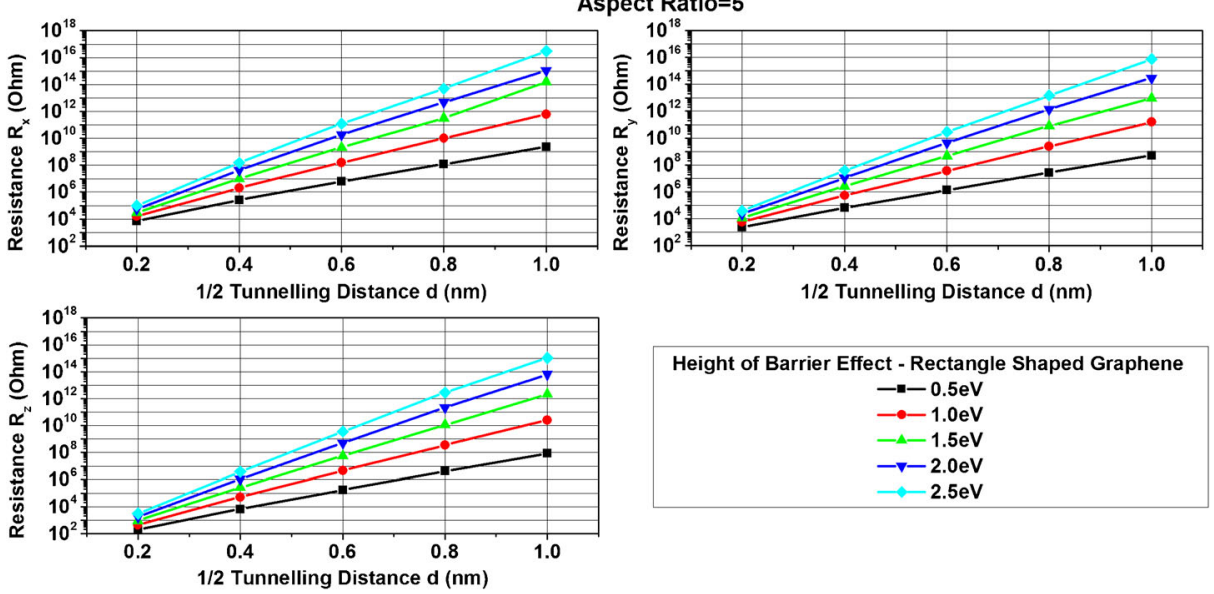

\begin{tabular}{|c|}
\hline Height of Barrier Effect - Rectangle Shaped Graphene \\
$--0.5 \mathrm{eV}$ \\
$--1.0 \mathrm{eV}$ \\
$--1.5 \mathrm{eV}$ \\
$-\checkmark-2.0 \mathrm{eV}$ \\
$-\leftarrow-2.5 \mathrm{eV}$
\end{tabular}

Fig. 6 Effect of height of barrier (energy state of the insulating material to be reached by the electrons so as to conduction occur) on unit cell resistance for the case of rectangular shaped graphene filler and aspect ratio of $1 \&$ 5 in respect of the direction and the tunnelling resistance

network in the volume of nanocomposite at lower volume fraction. The above conclusion is supported also by the fact that with the rise of volume fraction the deviation on the statistical sample is significantly reduced leading to more uniform sample close to uniquely defined electrical conductivity for a nanocomposite of a specific volume fraction [36].

In Fig. 9, the effect of the height of barrier on the electrical conductivity is depicted for the case of rectangle shaped graphene with aspect ratio equal to 1 and 5. Although, the percolation

Fig. 7 Convergence study on the size of the representative volume for the case of the graphene filler with aspect ratio $=1$ and $\mathrm{V}_{\mathrm{f}}=0.4$

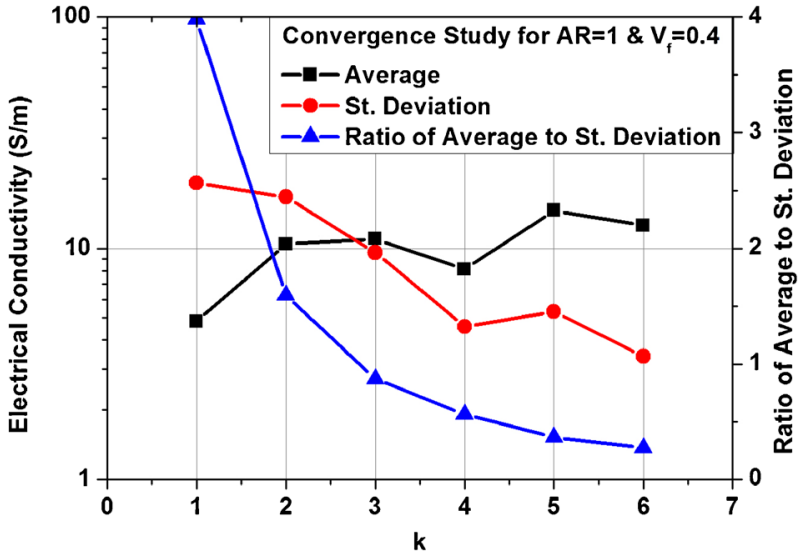


Fig. 8 Percolation probability in respect of volume fraction for studied graphene aspect ratios for the case of $\lambda=0.5 \mathrm{eV}$ and the corresponding functions

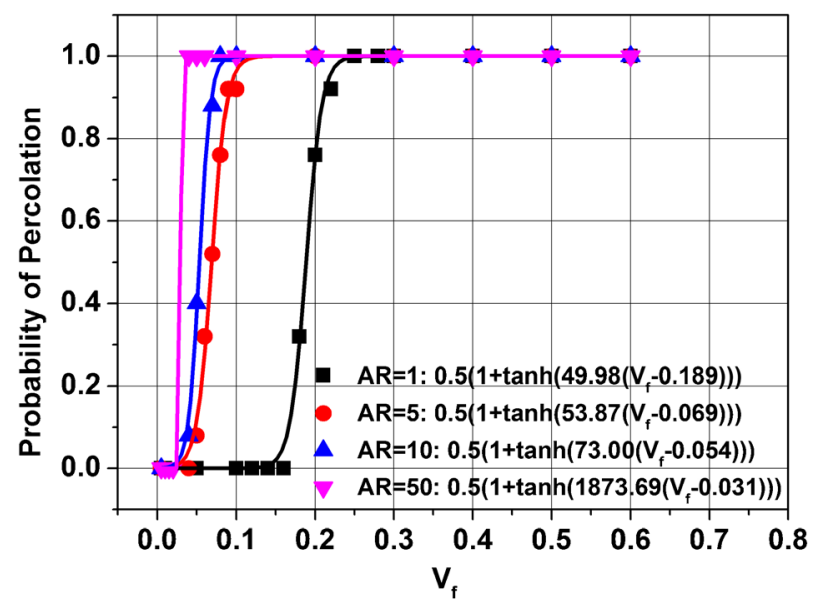

threshold seems not to be affected by the height of barrier, since the onset of percolation is mainly geometrically oriented as it has been proven by [25]. For the case of constant volume fraction, the rise of the height of barrier causes rapid decrease on the electrical conductivity even by a decrease of an order of magnitude in the case of increasing the height of barrier by $0.5 \mathrm{eV}$.

Finally, the effect of the graphene shape is calculated in respect of the volume fraction for the case of height of barrier being $0.5 \mathrm{eV}$ [36]. The graphene shape does not affect the trend of the nanocomposite's electrical response in function of the volume fraction $\left(\mathrm{V}_{\mathrm{f}}\right)$. Although for graphene particles of the same aspect ratio and volume, the nanocomposite reinforced with rectangle shaped particles exhibits higher electrical conductivity than the one of the ellipse shaped, while the rectangle shaped graphene particles show comparable percolation threshold to that of the ellipse graphene. Yi et al. [37] suggested that this observation could be explained as the corner angles of squares and rectangles make it easier for the plates to touch each other, therefore enhancing the current passing from one particle to another.

\subsection{Percolation Model}

For the description of a nanocomposite's electrical behaviour, there are two main theories being suggested, the percolation law -which describes the variation of the electrical
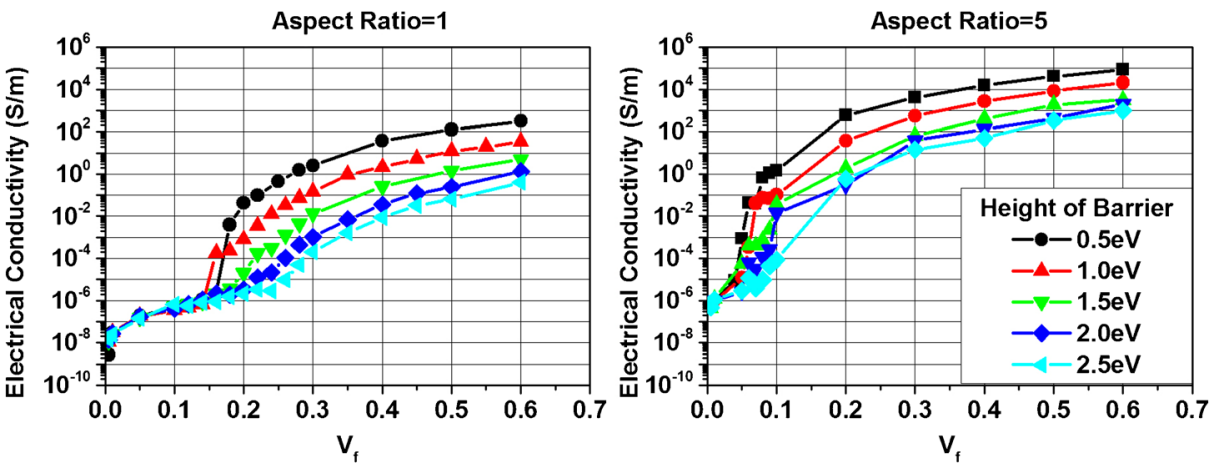

Fig. 9 Effect of height of barrier on the electrical conductivity in respect of volume fraction $V_{f}$ for rectangle shaped graphene and aspect ratios of $1 \& 5$ 
conductivity of the nanocomposite in function of the volume fraction after the percolation threshold having being obtained- and the excluded volume theory-which predicts the percolation threshold in respect of the geometrical features of the reinforcement (dimensions, shape, 2D-3D conductive networks).

Taking into consideration the excluded volume theory and assuming that each particle is considered equivalent to a circular one, the excluded volume theory for the circular disk [38] could be modified to define the upper and lower boundaries of the volume fraction in which the percolation threshold should be expected (Eq. 15). In Fig. 10, it is obvious that the percolation threshold is reduced with increasing aspect ratio, the values were successfully found to lie between the assumed percolation bounds. Moreover, the shape of the graphene does not show any effect on the percolation threshold, since the corresponding values for each case are close enough to consider any intense effect of the filler's shape.

$$
\begin{aligned}
& 1-\exp \left(-1.4 \frac{V}{V_{e x}}\right) \leq V_{c} \leq 1-\exp \left(-2.8 \frac{V}{V_{e x}}\right) \\
& V=\pi\left(\frac{d}{2}\right)^{2} t \\
& V_{e x}=\pi^{2}\left(\frac{d}{2}\right)^{3} \\
& \left\{\stackrel{\frac{d}{2}-a \sqrt{\frac{A R}{\pi}}}{\rightarrow} 1-\exp \left(-1.4 t \frac{\sqrt{\pi}}{a \sqrt{A R}}\right) \leq V_{c} \leq 1-\exp \left(-2.8 t \frac{\sqrt{\pi}}{a \sqrt{A R}}\right)\right.
\end{aligned}
$$

The percolation law is a power equation (16) introduced in [39], which is able to describe to electrical behaviour of an insulating material reinforced with conductive particles after the percolation threshold. In equation (16), $\sigma_{c}$ is the electrical conductivity of the composite, $V_{f}$ is the volume fraction, $V_{p}$ is the percolation threshold, $\sigma_{o}$ is a factor usually calculated to be close to the electrical conductivity of the conductive phase and $t$ is the critical exponent. For a three dimensional conductive network, $t$ usually takes value between 1.65 and 2.0, which is accepted as a universal value [39]. Higher values of $t$ indicate the presence of tunnelling phenomena and the filler having extreme geometries (i.e. high aspect ratio) [39]. In Fig. 11, the fitting of the finite element model results in accordance to the percolation law is presented, showing a good correlation. Figure 12 depicted the effect of the geometry of the filler, and the height of barrier on the percolation law variables. $\sigma_{\mathrm{o}}$ increases with rising aspect ratio

Fig. 10 Percolation threshold in respect of the graphene shape and boundary functions (15)

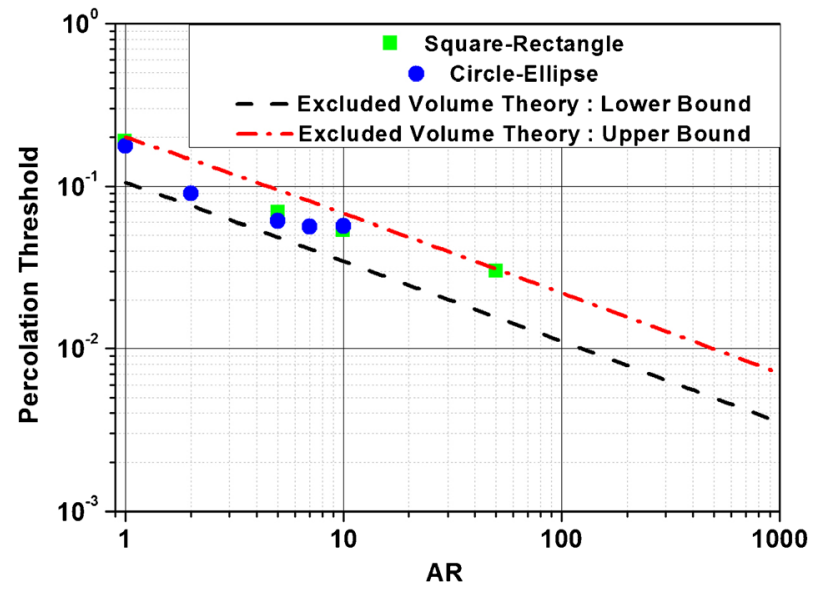


converging to the filler electrical conductivity, while it decreases with increasing height of barrier. The increase of the aspect ratio leads to more stable electrical networks which are mainly governed by the fillers conductance and not the tunnelling resistance formed in the interparticle region, leading $\sigma_{\mathrm{o}}$ to converge to the electrical conductivity of the filler. On the other hand, the rise of the height of barrier which induces a rise on the tunnelling resistance in the interparticle volume has as a consequence the decrease of the $\sigma_{\mathrm{o}}$ for the case of low aspect ratios. One of the most significant findings related to the percolation law is the agreement of the observation to the general law regarding the critical exponent. An increase of the aspect ratio (defining an extreme geometry) or even any rise of the height of barrier (defining a more intense tunnelling phenomenon) has a result an important increase of the value of the critical exponent far above the prediction for a common three dimensional conductive network.

$$
\sigma_{c}=\sigma_{o}\left(V_{f}-V_{p}\right)^{t}
$$

From Fig. 12, some important notices could be extracted regarding the development of an effective design tool for preparing appropriate material structures to serve the specified needs of a certain application. First of all, it could be concluded that Fig. 12 could be characterised as constitution curves, since the effect of the each variable (height of barrier, geometry and filler type) on the electrical response of the material could be depicted in a simple and easily understood manner, in respect of the percolation law parameters. These easily produced graphs could be used as a design tool for effective manufacturing nano-reinforced materials with desired electrical properties. Knowing the effect of several geometrical, material and manufacturing parameters on the final electrical performance of the nanocomposite in terms of the variation of percolation law variables, the number of "trial \& error" procedures are reduced to the necessary ones to control the material system characteristics so as to produce a conductive nanocomposite with electrical response comparable to the one expected from the numerical analysis.

\subsection{Preliminary Model Validation on the Prediction of the Percolation Threshold}

The aforementioned methodology is employed on experimental material results found in literature for the prediction of the percolation threshold of these materials and their possibility
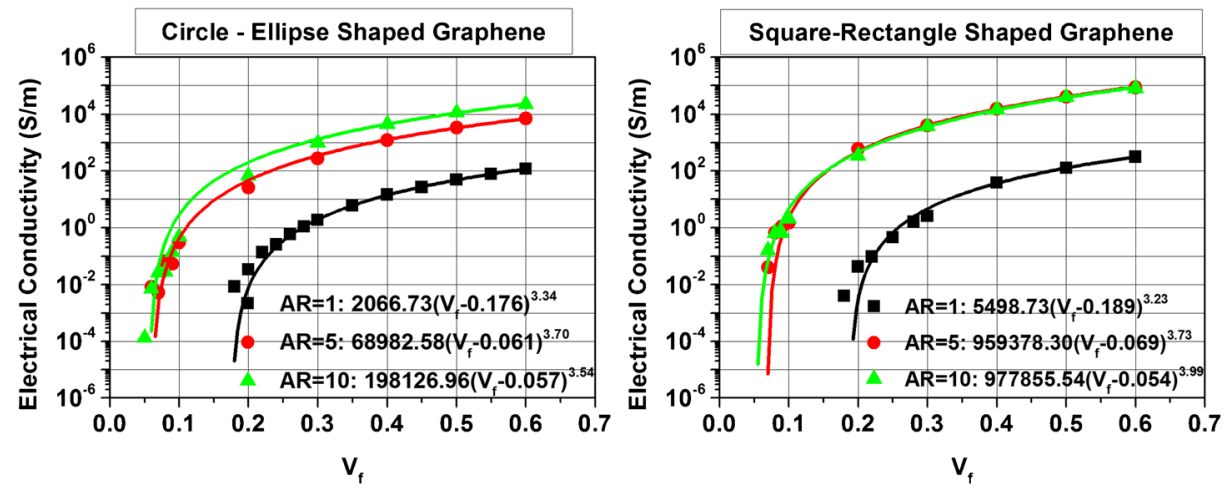

Fig. 11 Percolation Law in respect of the aspect ratio and the shape of the graphene particle for the case of height of barrier of $0.5 \mathrm{eV}$ 

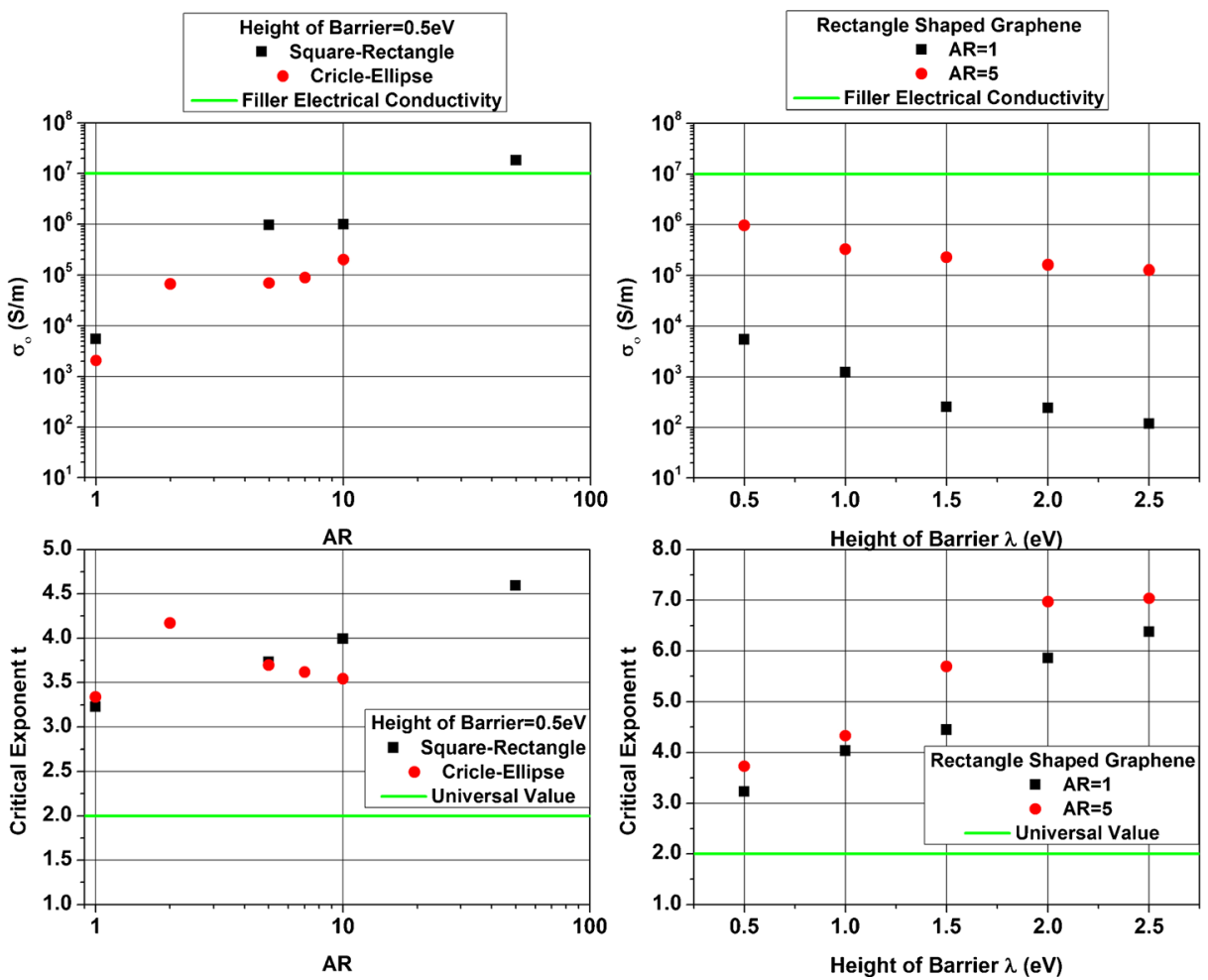

Fig. 12 Variation of the percolation law variables in respect of the aspect ratio, the shape of graphene and the height of barrier

of conductance under given circumstances was calculated. At first, the methodology is employed for the material structure of Poly(vinylidene fluoride)/Graphene proposed in the experimental work of $\mathrm{Yu}$ et al. [40]. In this work, PVDF/Graphene nanocomposite was prepared by adopting a solution method. The graphene nanosheets exhibited an average thickness around $1 \mathrm{~nm}$, while the particle size range from 0.1-7 $\mu \mathrm{m}$ with mean diameter around $2 \mu \mathrm{m}$ as it could be calculated in the Fig. 1c quoted in the work [40]. Two cases of reinforcement with constant thickness of $1 \mathrm{~nm}$ - minimum diameter of $0.1 \mu \mathrm{m}$ and average diameter of $2 \mu \mathrm{m}$ and random distribution of the filler in the polymer bulk volume are modelled. The percolation threshold is calculated for each case as well as the percolation probability. It could be easily noted by Fig. 13, that the experimental value of percolation threshold (4.5 wt $\%)$ is well predicted by assuming the average value of the filler size distribution. To predict the percolation threshold of a graphene based nanocomposites, it is not necessary to simulate the size distribution of the filler, but just to use the average value of this.

Since, the percolation threshold is not only highly dependent on the particle size but on the preparation process which affect the filler distribution on the bulk volume of the polymer, the effect of the manufacturing process should be taken into account. When a nanocomposite is manufactured with a solution method, it could be sufficiently assumed that the distribution of the filler is random. On the other hand, if the material was prepared with solution casting of films which were then stacked layer by layer during a hot-press process, it could be assumed that the in-plane filler distribution could be random due to the solution casting of the films, 
Fig. 13 Prediction of the percolation threshold for the material structure proposed on the experimental work of $\mathrm{J}$. Yu et al. [40]

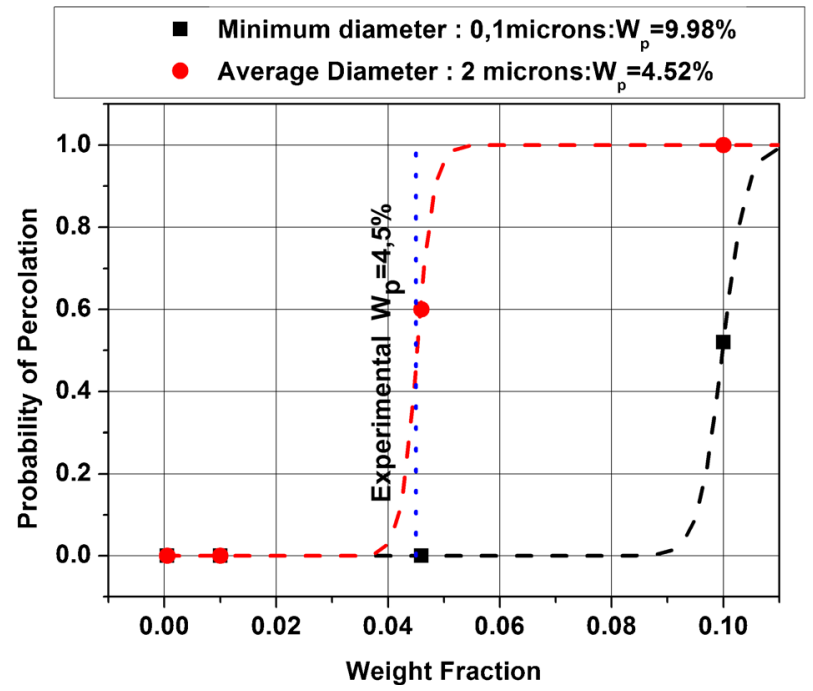

whereas a through-the-thickness Gaussian distribution could be assumed due to the hotpressing of the films. These assumptions are used for the prediction of the percolation threshold of the Poly(vinylidene fluoride)/Exfoliated Graphite nanoplatelets suggested in the experimental work of He et al. [41], while they were supported by the SEM image of the fractured cross-section of $\mathrm{PVDF} / \mathrm{xGnP}$ nanocomposite with $\mathrm{V}_{\mathrm{f}}=0.76 \mathrm{vol} \%$ showing nanoplatelets being concentrated in the middle of the specimen. Since, in the experimental work there was not any experimental study on the particle distribution into the polymer volume, there was a parametrical study on the effect of the standard deviation assuming the average was constant and set in the middle of the specimen thickness. In accordance to the experimental work, the graphite platelets had a diameter of $25 \mu \mathrm{m}$ and thickness of $20 \mathrm{~nm}$ and these dimensions were introduced in the model. For reasons of comparison the same study is conducted with the randomly distributed filler, while the obtained percolation probability is compared to the one with the filler being normally distributed with an average $\mu=62,5 \mu \mathrm{m}$ and standard deviation $\sigma=20.8 \mu \mathrm{m}$ depicted in Fig. 14. The percolation threshold is $3.83 \mathrm{vol} \%$ for randomly distributed filler and 2.34 vol\% when the filler followed the Gaussian distribution proving a profound difference between these two modelling assumptions.

Authors stated that the percolation threshold of the proposed material structure was estimated to be $1.09 \mathrm{vol} \%$. As a consequence, the parametrical study on the effect of the standard deviation was made for a constant volume fraction of the experimentally obtained percolation threshold, so as to explore the effect of the standard deviation on the percolation probability. The results of this study are depicted in Fig. 15. For $\mathrm{V}_{\mathrm{f}}=1.09 \%$, the sample exhibits probability of conductance for standard deviation $\sigma$ lower than $12 \mu \mathrm{m}$, while the studied $\mathrm{V}_{\mathrm{f}}$ would be considered as percolation threshold of the nanocomposite (in correspondence to the definition of percolation threshold, it is the $\mathrm{V}_{\mathrm{f}}$ where the $50 \%$ of the sample is conductive, or the percolation probability is $50 \%$ )- if the $\sigma$ is $7.35 \mu \mathrm{m}$. Taking into consideration the above conclusions on the study of the percolation threshold of PVDF/ Exfoliated Graphite Nanoplates prepared by He et al. [41], the importance of studying statistical samples and calculating the percolation probability of each sample during experimental works is recognized. This feature should be included to electrical conductivity 
Fig. 14 Comparison between the percolation probability of the randomly and Gaussian distributed through-the-thickness filler

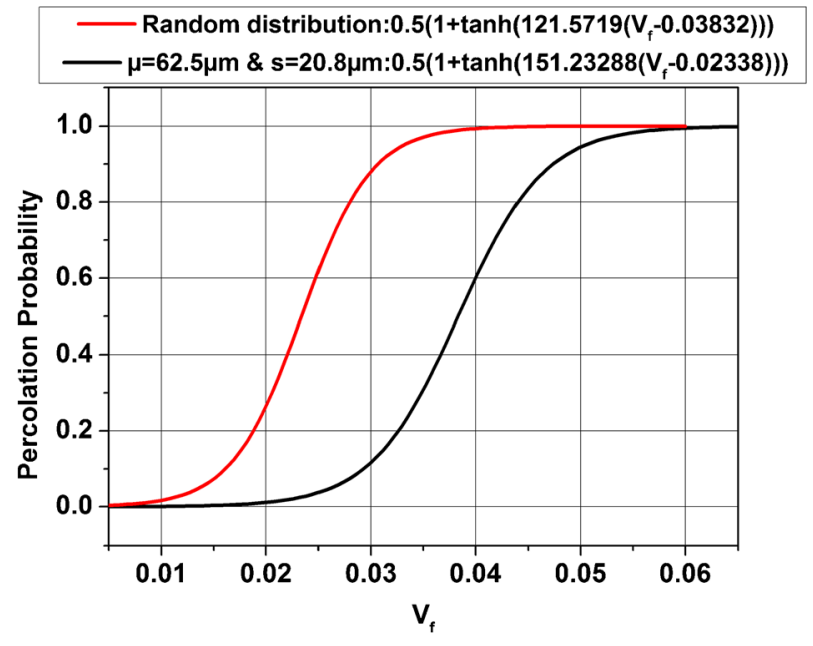

characterization quantities, so as to be fully possible to compare the effectiveness of different material manufacturing techniques, the electrical performance of similar material structures prepared by different research groups as well as to assist the validation process of suggested simulation methods. Moreover, the parametrical study on the effect of standard deviation on the percolation probability for a given value of $V_{f}$ with experimentally known percolation probability could be an additional tool on a nanocomposite's filler distribution characterization in conjunction with imaging techniques (SEM images mapping for instance).

\section{Concluding Remarks}

In literature, there are several graphene/polymer material systems suggested to be electrically and/ or even thermally conductive, however there are a few computational/analytical methods being

Fig. 15 Standard Deviation effect on the percolation probability for $\mathrm{V}_{\mathrm{f}}$ constant to the experimental percolation threshold found on the work [41] and average $\mu=62.5 \mu \mathrm{m}$

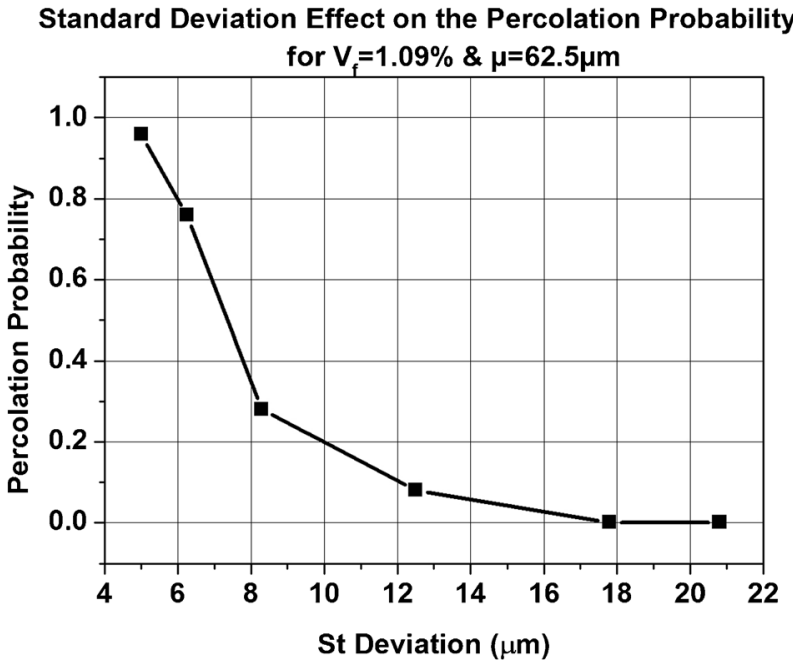


able to predict their full response. Most of them are geometry based and are able to predict the percolation threshold. In this paper, a parametrical multi-scale finite element model was proposed to simulate the full electrical response of graphene based polymer nanocomposites.

The multi-scale multi-physics finite element model was found to predict the electrical response of the selected graphene/polymer composite under DC loading, while the results are in accordance with theoretical predictions. It was proven that the increase of the aspect ratio reduces the percolation threshold and increases the electrical conductivity of the nanocomposite for a given value of volume fraction. The tunnelling resistance exhibited in the inter-particle volume affects the overall performance of the nanocomposite, especially due to the height of barrier, whose rise increases the inter-particle resistance and decreases the electrical conductivity of the nanocomposite. The shape of the graphene fillers do not show any significant effect in terms of percolation, but the formation of sufficient contact between particles for the charge transfer is enhanced for the case of rectangular shaped graphene. One of the most important findings of the parametrical analysis is the capability to create constitution curves by plotting the effect of each examined parameter on the variables of the percolation law. These graphs could be used as a preliminary design tool for more effective material system manufacturing.

Finally, the methodology was used in order to predict the percolation of threshold of two similar material systems whose electrical response was studied experimentally, prepared with different manufacturing process, so as to explore the effect of material preparation on the percolation threshold, the percolation probability and the adaptability of the proposed simulation methodology. The percolation threshold was calculated with important accuracy, exhibiting error of $0.44 \%$ of the analytical result compared to the experimental data, for the case of the PVDF/Graphene nanocomposite [40], while a study on the effect of the filler distribution on the percolation probability of $\mathrm{PVDF} / \mathrm{xGnP}$ nanocomposite [41] with volume fraction equal to the experimentally obtained percolation threshold was made, arising the significance of including the percolation probability as characterization quantity. It is suggested this model's application to be extended to the simulation of the electrical response of experimental case study nanocomposites, while the obtained behaviour should be related to the probability of achievement under real manufacturing and experimental conditions.

Open Access This article is distributed under the terms of the Creative Commons Attribution 4.0 International License (http://creativecommons.org/licenses/by/4.0/), which permits unrestricted use, distribution, and reproduction in any medium, provided you give appropriate credit to the original author(s) and the source, provide a link to the Creative Commons license, and indicate if changes were made.

\section{References}

1. Sichel, E.K., Gittleman, J.I., Sheng, P.: Electrical properties of carbon-polymer composites. J. Electron. Mater. 11, 699-747 (1982)

2. Ishigure, Y., Iijima, S., Ito, H., Ota, T., Unuma, H., Takahashi, M., Hikichi, Y., Suzuki, H.: Electrical and elastic properties of conductor-polymer composites. J. Mater. Sci. 34, 2979-2985 (1999)

3. Pinto, G., Jiménez-Martín, A.:Conducting aluminum-filled nylon 6 composites. Polym. Compos., 22, 65-70 (2001)

4. Roldughin, V.I.: Vysotskii, V. V.:percolation properties of metal-filled polymer films, structure and mechanisms of conductivity. Prog. Org. Coatings. 39, 81-100 (2000)

5. Flandin, L., Cavaillé, J.Y., Bidan, G., Brechet, Y.: New nanocomposite materials made of an insulating matrix and conducting fillers: Processing and properties. Polym. Compos. 21, 165-174 (2000)

6. Ray, S.S., Biswas, M.: Water-dispersible conducting nanocomposites of polyaniline and poly(Nvinylcarbazole) with nanodimensional zirconium dioxide. Synth. Met. 108, 231-236 (2000) 
7. Quivy, A., Deltour, R., Jansen, A.G.M.: Wyder, P.:transport phenomena in polymer-graphite composite materials. Phys. Rev. B. 39, 1026-1030 (1989)

8. Zheng, W., Wong, S.-C.: Sue, H.-J.:transport behavior of PMMA/expanded graphite nanocomposites. Polymer. 73, 6767-6773 (2002)

9. Zheng, W., Wong, S.-C.: Electrical conductivity and dielectric properties of PMMA/expanded graphite composites. Compos. Sci. Technol. 63, 225-235 (2003)

10. Chen, G., Weng, W., Wu, D.: Wu, C.:PMMA/graphite nanosheets composite and its conducting properties. Eur. Polym. J. 39, 2329-2335 (2003)

11. Kim, H., Miura, Y., Macosko, C.W.: Graphene/polyurethane nanocomposites for improved gas barrier and electrical conductivity. Chem. Mater. 22, 3441-3450 (2010)

12. Song, Y., Yu, J., Yu, L., Alam, F.E., Dai, W., Li, C., Jiang, N.: Enhancing the thermal, electrical, and mechanical properties of silicone rubber by addition of graphene nanoplatelets. Mater Design. 88, 950-957 (2015)

13. Yun, C., Feng, Y., Qiu, T., Yang, J., Li, X., Yu, L.: Mechanical, electrical, and thermal properties of graphene nanosheet/aluminum nitride composites. Ceram. Int. 41, 8643-8649 (2015)

14. Al-Saleh, M.H.: Electrical and mechanical properties of graphene/carbon nanotube hybrid nanocomposites. Synth. Met. 209, 41-46 (2015)

15. Kandare, E., Khatibi, A.A., Yoo, S., Wang, R., Ma, J., Olivier, P., Gleizes, N., Wang, C.H.: Improving the through-thickness thermal and electrical conductivity of carbon fibre/epoxy laminates by exploiting synergy between graphene and silver nano-inclusions. Compos. Part A. 69, 72-82 (2015)

16. Oskouyi, A.B., Mertiny, P.: Monte Carlo model for the study of percolation thresholds in composites filled with circular conductive nano-disks. Procedia Eng. 10, 403-408 (2011)

17. Ambrosetti, G., Johner, N., Grimaldi, C., Danani, A., Ryser, P.: Percolative properties of hard oblate ellipsoids of revolution with a soft shell. Phys. Rev. E. 78, 061126:1-061126:11 (2008)

18. Otten, R.H.J., Van Der Schoot, P.: Connectivity percolation of polydisperse anisotropic nanofillers. J. Chem. Phys. 134, 094902:1-094902:15 (2011)

19. Xia, W., Thorpe, M.F.: Percolation properties of random ellipses. Phys. Rev. A. 38(5), 2650-2656 (1988)

20. Vovchenko, L., Vovchenko, V.: Simulation of percolation threshold in composites filled with conducting particles of various morphologies. Materwiss. Werksttech. 42, 70-74 (2011)

21. Mathew, M., Schilling, T., Oettel, M.: Connectivity percolation in suspensions of hard platelets. Phys. Rev. E Stat. Nonlinear Soft Matter Phys. 85(061407), (2012)

22. Hicks, J., Behnam, A., Ural, A.: A computational study of tunneling-percolation electrical transport in graphene-based nanocomposites. Appl. Phys. Lett. 95, 213103:1-213103:3 (2009)

23. Ambrosetti, G., Grimaldi, C., Balberg, I., Maeder, T., Danani, A., Ryser, P.: Solution of the tunnelingpercolation problem in the nanocomposite regime. Phys. Rev. B. 81, 155434 (2010)

24. Oskouyi, A.B., Sundararaj, U., Mertiny, P.: Tunneling conductivity and Piezoresistivity of composites containing randomly dispersed conductive Nano-platelets. Materials. 7, 2501-2521 (2014)

25. Hashemi, R., Weng, G.J.: A theoretical treatment of graphene nanocomposites with percolation threshold, tunnelingassisted conductivity and microcapacitor effect in AC and DC electrical settings. Carbon. 96, 474-490 (2016)

26. Simmons, J.G.: Generalized formula for the electric tunnel effect between similar electrodes separated by a thin insulating film. J. Apllied Phys. 34(6), 1793-1803 (1963)

27. Novoselov, K.S., Geim, A.K., Morozov, S.V., Jiang, D., Zhang, Y., Dubonos, S.V., Grigorieva, I.V., Firsov, A.A.: Electric field effect in atomically thin carbon film. Science. 306, 666-669 (2004)

28. Chen, Z., Lin, Y.-M., Rooks, M.J., Avouris, P.: Graphene nano-ribbon electronics. Phys. E. 40, 228-232 (2007)

29. Sidorov, A.N., Yazdanpanah, M.M., Jalilian, R., Ouseph, P.J., Cohn, R.W., Sumanasekera, G.U.: Electrostatic deposition of graphene. Nanotechnology. 18, 135301 (2007)

30. Mechler, A., Kokavecz, J., Heszler, P., Lal, R.: Surface energy maps of nanostructures: atomic force microscopy and numerical simulation study. Appl. Phys. Lett. 82, 3740 (2003)

31. Mechler, Á., Kopniczky, J., Kokavecz, J., Hoel, A., Granqvist, C.-G., Heszler, P.: Anomalies in nanostructure size measurements by AFM. Phys. Rev. B. 72, 125407 (2005)

32. Kühle, A., Sorensen, A.H., Zandbergen, J.B., Bohr, J.: Contrast artifacts in tapping tip atomic force microscopy. Appl. Phys. A Mater. Sci. Process. 66, S329-S332 (1998)

33. Gupta, A., Chen, G., Joshi, P., Tadigadapa, S., Eklund, P.C.: Raman scattering from high-frequency phonons in supported n-graphene layer films. Nano Lett. 6, 2667-2673 (2006)

34. Casiraghi, C., Hartschuh, A., Lidorikis, E., Qian, H., Harutyunyan, H., Gokus, T., Novoselov, K.S., Ferrari, A.C.: Rayleigh imaging of graphene and graphene layers. Nano Lett. 7, 2711-2717 (2007)

35. Nemes-Incze, P., Osváth, Z., Kamarás, K., Biró, L.P.: Anomalies in thickness measurements of graphene and few layer graphite crystals by tapping mode atomic force microscopy. Carbon. 46, 1435-1442 (2008)

36. Manta, A., Gresil, M., Soutis, C.: Multi-scale finite element analysis of graphene/polymer nanocomposites: electrical Perfomance. VII European Congress on Computational Methods in Applied Sciences and Engineering (2016) 
37. Yi, Y.B., Tawerghi, E.: Geometric percolation thresholds of interpenetrating plates in three-dimensional space. Phys. Rev. E. 79, 041134:1-041134:6 (2009)

38. Balberg, I., Anderson, C.H., Alexander, S., Wagner, N.: Excluded volume and its relation to the onset of percolation. Phys. Rev. B. 30, 3933-3943 (1984)

39. Stauffer, D., Aharony, A.: Introduction to Percolation Theory. Taylor \& Francis, London (1994)

40. Yu, J., Huang, X., Wu, C., Jiang, P.: Permittivity, thermal conductivity and thermal stability of poly(vinylidene fluoride)/graphene nanocomposites. IEEE Trans. Dielectr. Electr. Insul. 18, 478-484 (2011)

41. He, F., Lau, S., Chan, H.L., Fan, J.: High dielectric permittivity and low percolation threshold in nanocomposites based on poly(vinylidene fluoride) and exfoliated graphite Nanoplates. Adv. Mater. 21, $710-715$ (2009) 